Boletín de la Sociedad Geológica MeXicana, TOMO LV, NúM. 1, 2002, P. 1-11

\title{
Análisis estructural de fallas potencialmente activas
}

\author{
Odranoel Quintero-Legorreta ${ }^{\dagger}$
}

Instituto de Geología,

Universidad Nacional Autónoma de México, Cd. Universitaria, 04510 México, D.F.

\begin{abstract}
Resumen
El análisis estructural de fallas potencialmente activas comprende el estudio de las deformaciones recientes y está relacionado con la aplicación de los métodos y fines de la neotectónica. Esta disciplina aplica métodos propios al establecimiento de una cronología fina de las deformaciones actuales en la corteza superior, siempre asociados a eventos tectónicos. En este trabajo se presentan algunos métodos que se utilizan para el estudio de las fallas activas y para ilustrarlo se utilizaron ejemplos en la parte central de Faja Volcánica Transmexicana. En particular, se destaca el papel que juegan en la geotectónica las estructuras regionales, simples y menores. Además, se presentan algunos métodos para el cálculo de los tensores de esfuerzo a partir de datos de fallas.

Palabras clave: Neotectónica, fallas normales, fallas activas, paleo-esfuerzos, Faja Volcánica Transmexicana.
\end{abstract}

\begin{abstract}
The structural analysis of potentially active faults encompasses the study of recent deformation and is the subject of neotectonics. This discipline apply specific methods to establish the chronology of recent deformation events in the upper crust and its relation to tectonics. In this work, the most common methods used in the study of active faults are presented, and examples from the central part of the Transmexican Volcanic Belt are presented in order to illustrate these methods. Particularly, the role of regional, simple and minor structures in geotectonics is emphasized. Some methods used for the calculation of stress tensors from data of faults are also presented.
\end{abstract}

Keywords: Neotectonics, normal faults, active faults, paleo-stress, Transmexican Volcanic Belt.

\section{Características generales de los métodos utilizados en neotectónica}

Los eventos neotectónicos pueden estudiarse tomando como referencia sus manifestaciones tectónicas, sedimentológicas, volcánicas y geofísicas.

Las deformaciones tectónicas afectan esencialmente a los bordes de las placas y, en mucho menor grado, el interior de las mismas. Esas deformaciones son el producto de los desplazamientos horizontales y verticales de las placas, las cuales se mueven de acuerdo con una cinemática conocida.
Los depósitos de las cuencas sedimentarias situadas en el interior o en la periferia de las placas litosféricas, registran de manera muy precisa los movimientos verticales (e.g. hundimientos, levantamientos), lo cual permite conocer la componente vertical de la deformación. La cronología de los eventos tectónicos queda registrada en los depósitos sedimentarios, pero su naturaleza (extensión-contracción-cizallamiento) queda por determinar.

Las emisiones volcánicas, y de manera más general el magmatismo, son producto de los eventos de deformación; éstos pueden ser fechados con la ayuda de los métodos isotópicos. Además, la composición química y 
mineralógica de las rocas resultantes son determinantes para conocer el ambiente tectónico en que se originaron los magmas.

En relación con los métodos geofísicos, las anomalías magnéticas del fondo oceánico nos proporcionan información sobre los cambios en la velocidad de los movimientos tectónicos en cierto tiempo. Por otro lado, la actividad sísmica es una expresión de las deformaciones tectónicas actuales; los perfiles sísmicos de reflexión permiten seguir en el mar los accidentes tectónicos conocidos en tierra. Los mecanismos focales y los perfiles sísmicos son herramientas que nos permiten evaluar las características fundamentales de las deformaciones recientes.

El estudio de los eventos neotectónicos puede ser abordado por vías diversas tales como la tectónica, la petrología, la estratigrafía, la sedimentología, la geomorfología y la geofísica. Por consiguiente, la neotectónica necesita de la estrecha cooperación de numerosas disciplinas. Para los fines de este artículo, se presentan solamente los métodos de análisis tectónico de las deformaciones recientes, los cuales siguen dos caminos.

El primer camino comprende el aspecto cronológico. Se trata de establecer la naturaleza de las deformaciones y su cronología para determinar las relaciones temporales entre los eventos tectónicos que afectan a las diferentes partes de una placa litosférica. Este estudio se hace por medio del análisis de las estructuras de escala regional; es necesario confrontar los eventos tectónicos con relación a los marcadores estratigráficos, geomorfológicos o volcánicos, y luego fechar esos marcadores estratigráficos por su cronología relativa o absoluta.

El segundo camino concierne al comportamiento mecánico de los materiales. Se trata de establecer, a partir de estudios tectónicos cuantitativos, los modelos de comportamiento mecánico de los materiales en superficie. El estudio de las estructuras menores (también conocido como microtectónica) es entonces muy importante. Los datos históricos de la actividad sísmica permiten establecer el enlace entre los resultados obtenidos para las deformaciones geológicas más recientes y aquellas obtenidas por la sismología instrumental (mecanismos focales de sismos) para las deformaciones actuales.

\section{Estructuras regionales}

Para ilustrar este trabajo he seleccionado ejemplos de la Faja Volcánica Transmexicana (FVTM). Este cinturón volcánico está localizado en la porción centro meridional de México y está constituido por distintos conjuntos volcánicos que fueron originados por procesos de subducción y de desgarre continental.

Los trabajos de análisis estructural en la FVTM se han desarrollado en las porciones central y occidental, localizadas entre la región de Acambay, Estado de México y Penjamillo, Guanajuato (Figura 1).

La parte central de la FVTM fue dividida en tres segmentos (Figura 2). El sector oriental está formado por el graben de Acambay; sobre esta estructura se registró un sismo de magnitud Ms=6.9 el 19 de noviembre de 1912. Este sismo ocasionó una falla de aproximadamente $52 \mathrm{~km}$ de largo y desplazamientos verticales de $50 \mathrm{~cm}$ en promedio a lo largo de las fallas normales que flanquean el graben (Urbina y Camacho, 1913). La pared norte de este graben está formada por relieves que varían en altura entre 300 y $400 \mathrm{~m}$ y la falla normal que la limita es la Falla de Acambay-Tixmadejé. La pared meridional está formada por relieves que se elevan 150 $\mathrm{m}$ en promedio a partir del fondo de la fosa tectónica. La falla normal que limita esta pared meridional es la Falla de Pastores.

El límite entre el sector oriental y el sector central está definido por una zona de transferencia o relevo entre

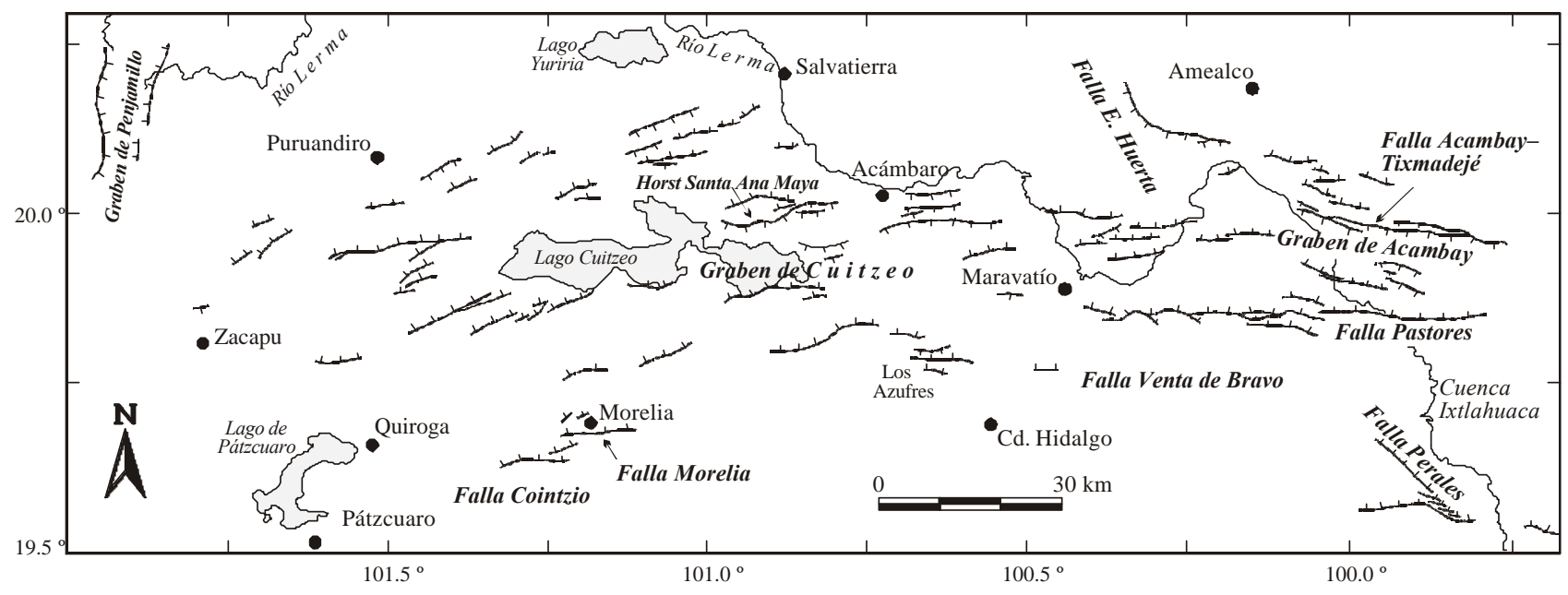

Figura 1. Patrón de fallas normales cuaternarias ubicadas en la parte central de la Faja Volcánica Transmexicana (modificado de Suter et al., 2001). 


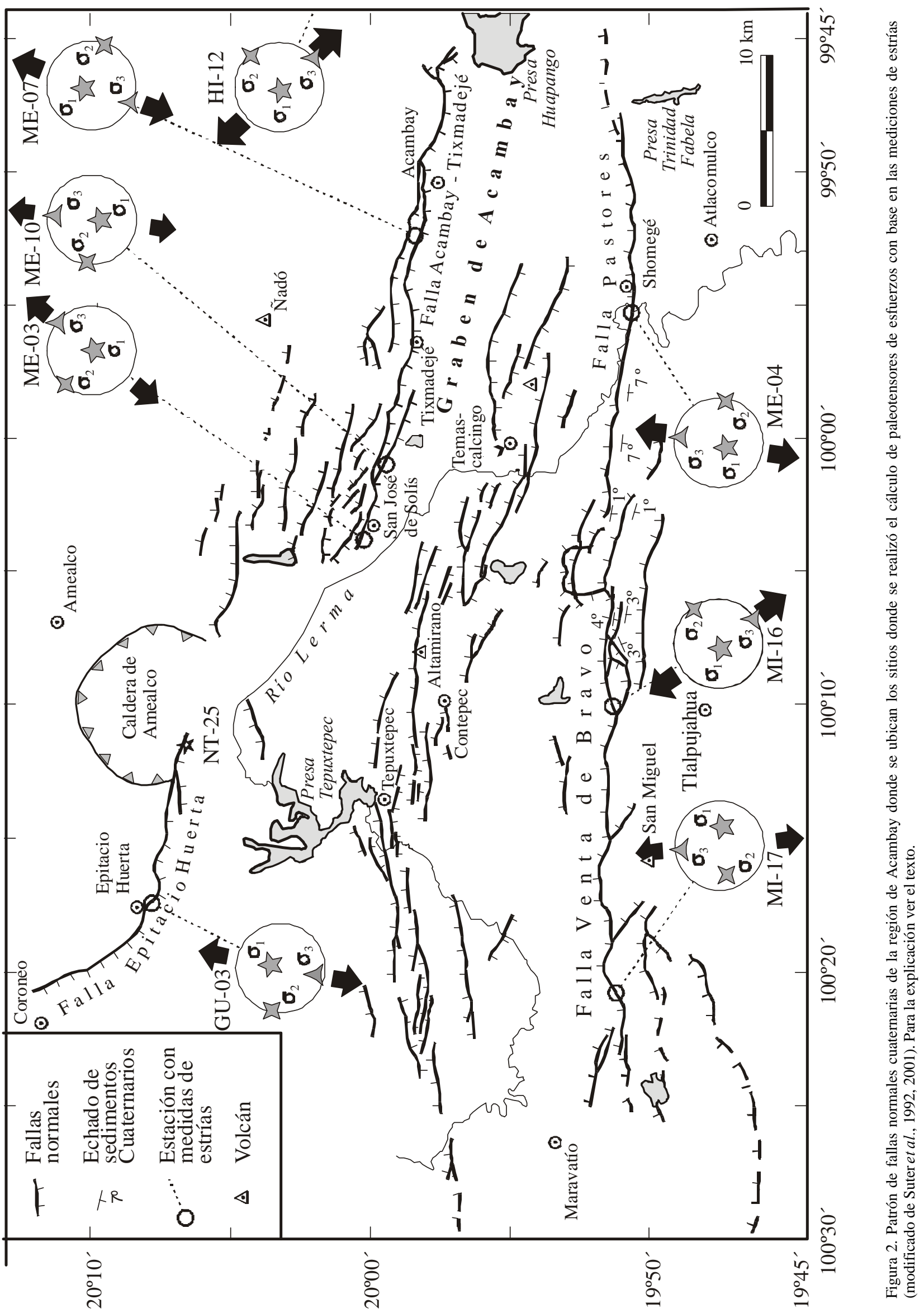


las fallas normales de Pastores y de Venta de Bravo, un cambio de $180^{\circ}$ en la dirección de deslizamiento de las fallas normales que afectan a la Caldera de Amealco y un cambio en la orientación del eje de la depresión del graben de Acambay con respecto a la llanura de Contepec (Figura 2). Estas tres características estructurales se alinean en la proyección hacia el sur de un lineamiento NNW-SSE denominado sistema de fallas Querétaro-Taxco.

El sector central se extiende entre el volcán Altamirano hacia el Este y la ciudad de Maravatío al Oeste. La estructura más sobresaliente de este sector es la falla de Venta de Bravo (Figura 2), la cual es una estructura sísmicamente activa que se extiende a lo largo de $45 \mathrm{~km}$. La terminación occidental de este segmento coincide con la terminación oriental del Graben de Cuitzeo, en una zona topográfica baja y llana donde se asienta la ciudad de Maravatío; en este sitio se ubica un rasgo Basin and Range alineado al NW-SE. La altura de los escarpes de falla dentro de este segmento es de $50 \mathrm{~m}$, con excepción de los escarpes de la falla principal donde el salto es mayor que $300 \mathrm{~m}$.

El sector occidental está definido por el área comprendida entre Morelia y Los Azufres; se caracteriza por fallas normales de orientación general E-W pertenecientes al sistema Morelia-Maravatío-Acambay (Figura 1). Algunas de las fallas rebasan los $25 \mathrm{~km}$ de longitud y la altura promedio de los escarpes es menor que $100 \mathrm{~m}$. Las fallas definen: 1) una depresión central que corresponde al Graben de Cuitzeo (Figura 1), la cual puede ser trazada desde la región oriental de la laguna de Cuitzeo hasta la región situada al sur de la Presa Solís; 2) un alto estructural localizado hacia el norte de la laguna de Cuitzeo y que corresponde al horst de Santa Ana Maya, el cual tiene un relieve topográfico de más de $850 \mathrm{~m}$ sobre el nivel de la Laguna de Cuitzeo; 3) un pequeño graben (Figura 1) al interior de la zona volcánica de Los Azufres (Dobson y Mahood, 1985).

Además del sistema de fallas E-W, existe un sistema

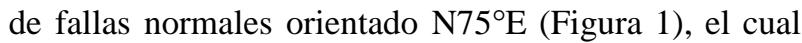
presenta escarpes pronunciados de 50 a $100 \mathrm{~m}$ de altura.

La zona volcánica de Los Azufres está cortada por un sistema de fallas normales E-W. Las rocas volcánicas mas jóvenes están fechadas en 0.15 Ma (Dobson y Mahood, 1985). Asociadas a las estructuras se registraron sismos locales cerca de Araró el 15 de mayo de 1845 (Arreola, 1985), y en la región de UcareoZinapécuaro-Acámbaro en octubre de 1872 y noviembre de 1874 (Orozco y Berra, 1887; Urquiza, 1872; Ramírez y Reyes, 1873).

\section{Estructuras simples}

Se trata de fallas de juego simple que han estado activas durante un período relativamente breve o bien que continúan activas. Estas estructuras han registrado en su historia tectónica un solo evento de deformación, que dio como resultado planos de falla con estrías en una sola dirección.

\subsection{Las fallas de juego simple en extensión}

Las fallas de juego simple en extensión son numerosas pero las más importantes son la Falla de AcambayTixmadejé, la Falla Pastores y la Falla Venta de Bravo; son fallas normales que se originaron bajo el mismo campo de esfuerzos regional.

\subsubsection{Falla de Acambay-Tixmadejé}

La Falla de Acambay-Tixmadejé forma el límite septentrional de un graben y presenta una longitud de aproximadamente $40 \mathrm{~km}$. Los escarpes que forman el bloque levantado alcanzan alturas de $400 \mathrm{~m}$ con respecto a la parte central del graben. El plano de falla presenta echados de $60^{\circ}$ y $70^{\circ}$ hacia el sur. El sitio ME-03 (Figura 2) fue estudiado cerca del poblado de San José de Solís. Los tensores calculados a partir de la población de fallas medidas en los sitios GU-03, ME-03, ME-10 y ME-07 muestran que esta falla se formó bajo una extensión orientada NE-SW (Figuras 2 y 3 ).

La falla activada por el sismo de 1912 se localiza al pie del escarpe de la falla de Acambay (Urbina y Camacho, 1913), un kilómetro al norte de Acambay. Esta fractura del terreno está ubicada en las orillas del poblado de Tixmadejé y tuvo un desplazamiento de 50 $\mathrm{cm}$. Este desplazamiento fue mayor al registrado en Acambay, donde varió entre 17 y $20 \mathrm{~cm}$. Hacia el Este, la falla ocasionó desplazamientos de $15 \mathrm{~cm}$. En el extremo occidental de esta falla la deformación cosísmica no fue reportada por Urbina y Camacho (1913) y la falla termina al occidente de San José de Solís, donde los escarpes son de menor altura con relación a la parte central. Este cambio sugiere que los desplazamientos mayores están en la parte central de la falla, siendo menores en los extremos de la misma.

\subsubsection{Falla de Pastores}

La Falla de Pastores forma la pared meridional del Graben de Acambay. Esta falla afecta a rocas volcánicas del Mioceno y del Cuaternario. A lo largo de la Falla de Pastores, Urbina y Camacho (1913) cartografiaron la zona de ruptura superficial que fue producida por el sismo de 1912. Ésta es una estructura de $17 \mathrm{~km}$ de longitud $\mathrm{y}$, aunque no se especificaron los desplazamientos, ellos mencionan que son semejantes a los de la falla Acambay-Tixmadejé, de alrededor de 20 a $50 \mathrm{~cm}$. La dirección de extensión obtenida a través del cálculo del tensor de esfuerzos, indica que ésta es perpendicular a la traza de la falla (sitio ME-04 en Figuras 2 y 4).

La falla no continuó hacia el oeste debido probablemente a la transferencia que existe entre la Falla de Pastores y la Falla de Venta de Bravo. Otras rupturas cosísmicas fueron cartografiadas por Urbina y Camacho (1913) en la parte central del Graben de Acambay, cerca 

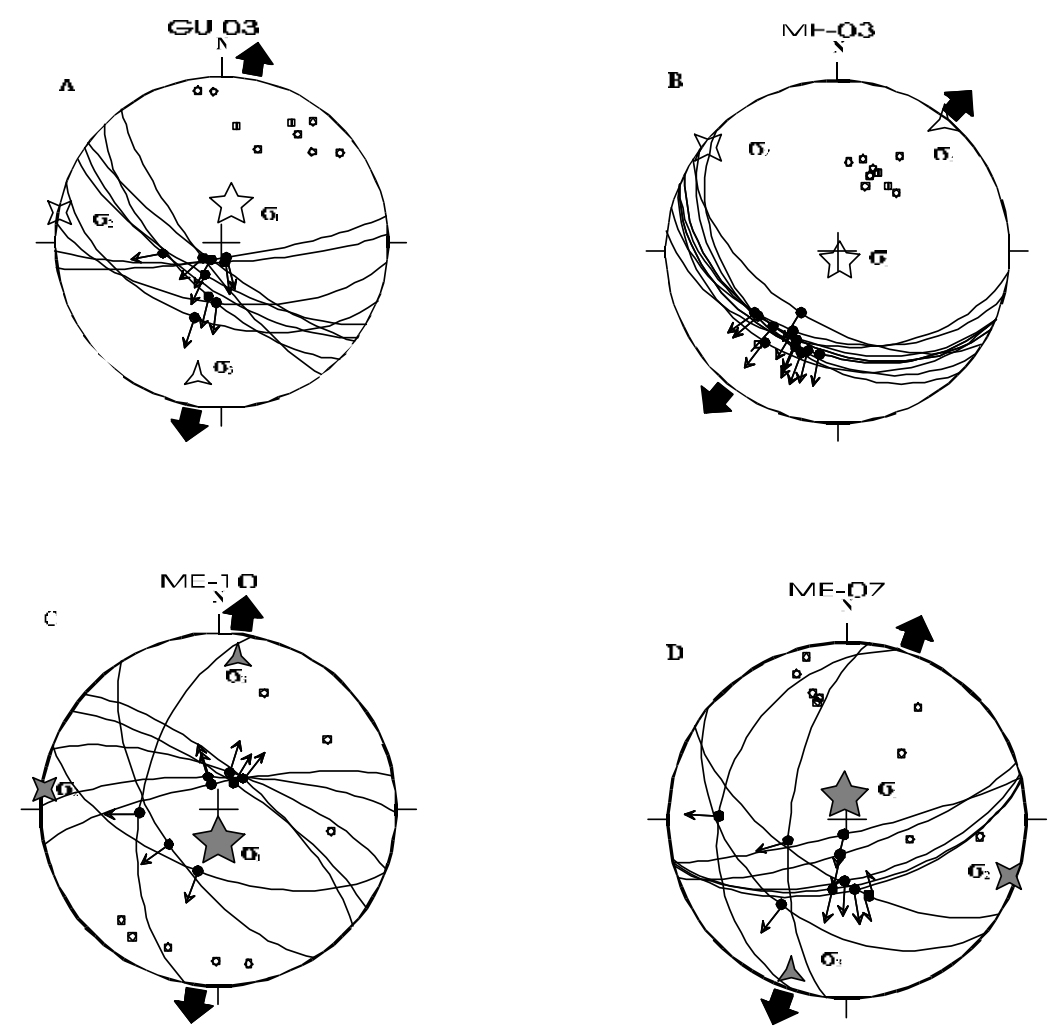

Figura 3. Diagramas estereográficos equiareales, de los tensores de esfuerzos calculados, mostrando los planos de fallas normales de juego simple en extensión y sus polos, así como sus estrías asociadas. Los sitios están localizados en la pared septentrional del Graben de Acambay.
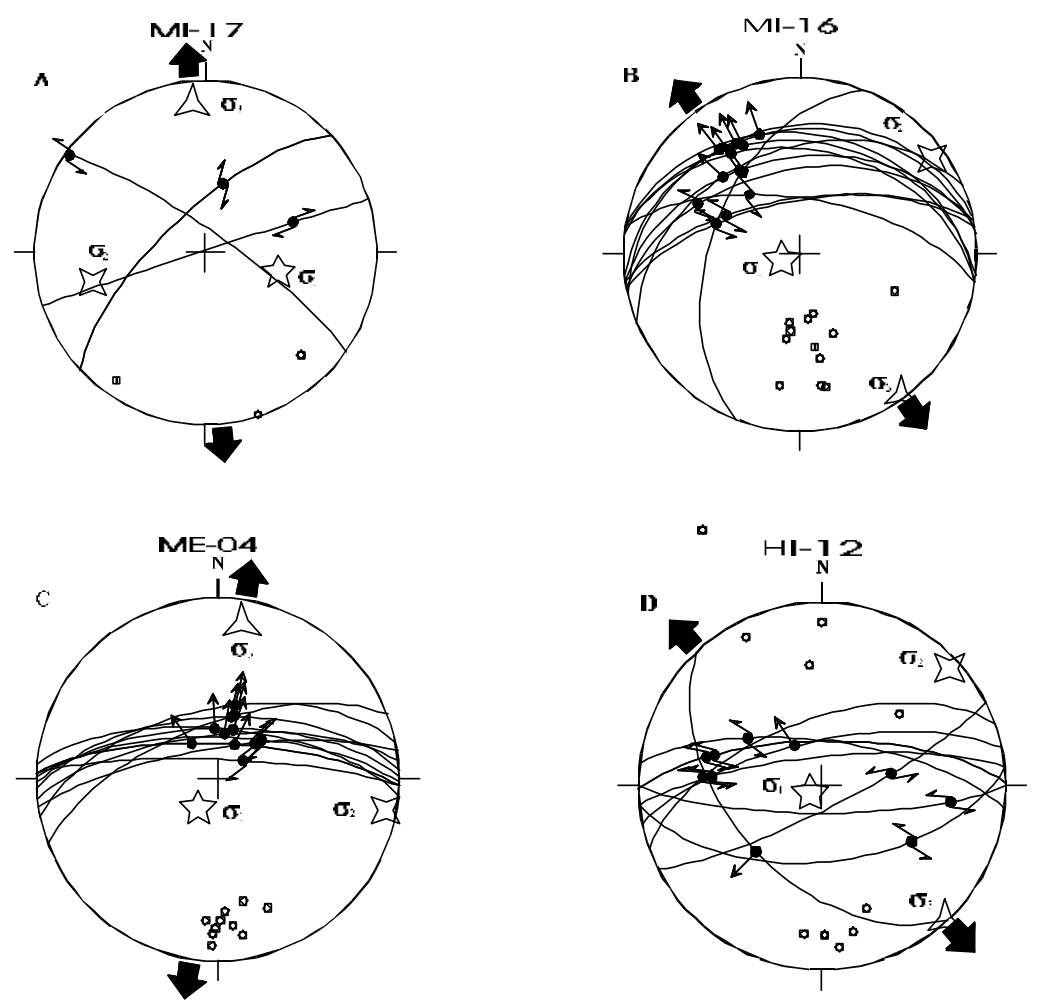

Figura 4. Diagramas estereográficos equiareales correspondientes al tensor de esfuerzos calculado, mostrando los planos de fallas normales de juego simple en extensión y sus polos así como sus estrías asociadas. Sitios localizados en la pared meridional del graben de Acambay. 
de la población de Temascalcingo. Estas estructuras se localizaron en la base de los múltiples escarpes de falla normal, cuyos desplazamientos son mayores que $30 \mathrm{~m} \mathrm{y}$ constituyen tres zonas de fractura paralelas orientadas E-W (Figura 2). La falla está orientada en general E-W a lo largo de $45 \mathrm{~km}$; el bloque hundido de esta falla se encuentra hacia el Norte, aunque existen segmentos de falla conectados a la falla principal en un arreglo en echelon. Al NNE de Tlalpujahua, la falla principal se ramifica definiendo un lente de $5 \mathrm{~km}$ de largo que internamente también está dislocado (Figura 2). La aparición de estas estructuras implica una componente de desplazamiento lateral izquierdo a lo largo de esta falla, aunque éste solamente está representado débilmente por los tensores de esfuerzo obtenidos en los sitios estudiados MI17 MI-16 ME-04 y HI-12 (Figuras 2 y 4). En lo que concierne al arreglo en echelon (Figura 2), éste corresponde a una etapa temprana de extensión oblicua lateral derecha, que después fue ligada por una extensión oblicua lateral izquierda y forma una etapa inicial de estructura pull-apart. También un movimiento menor de desplazamiento lateral izquierdo se interpreta a partir del mecanismo focal del sismo de Maravatío de 1979 (AstizDelgado, 1980) y del tensor calculado en el sitio MI-16, cercano al epicentro del sismo de 1979 (Figura 4).

Es necesario señalar que la observación de fallas de juego simple no implica necesariamente que la falla fue producida en su conjunto en un solo evento, ya que se pudo haber formado por movimientos sucesivos a través de cierto periodo de tiempo. En ocasiones ocurren por la reactivación de una falla más antigua en el sustrato. Así, por ejemplo, en el caso de las fallas de la región de Acambay, si existiera una falla más antigua originada por un régimen tectónico distinto del actual, la falla presentaría en su superficie diferentes familias de estrías debidas a la acción de distintos estados de esfuerzos. De esta manera la falla antigua presentaría estrías que correspondan a un juego simple en extensión pero superpuesto a uno quizá varios eventos más antiguos.

La simplicidad de movimiento en una falla, manifestada por un solo juego de estrías, es una suposición cómoda para el análisis de las fallas, ya que puede representar el último evento de deformación. Por lo tanto, las estructuras simples permiten reconocer fácilmente la naturaleza de las deformaciones (contracción o extensión) y, sobre todo, permiten separar en orden cronológico las etapas sucesivas de deformación.

\subsection{Fallas de juego simple en contracción.}

Para estudiar las características de las fallas en juego simple en contracción se eligieron dos áreas representativas, la región de la presa de Santa Rosa y la región de Hostotipaquillo, Jalisco (Figura 5).

En la región de la presa de Santa Rosa (al norte de

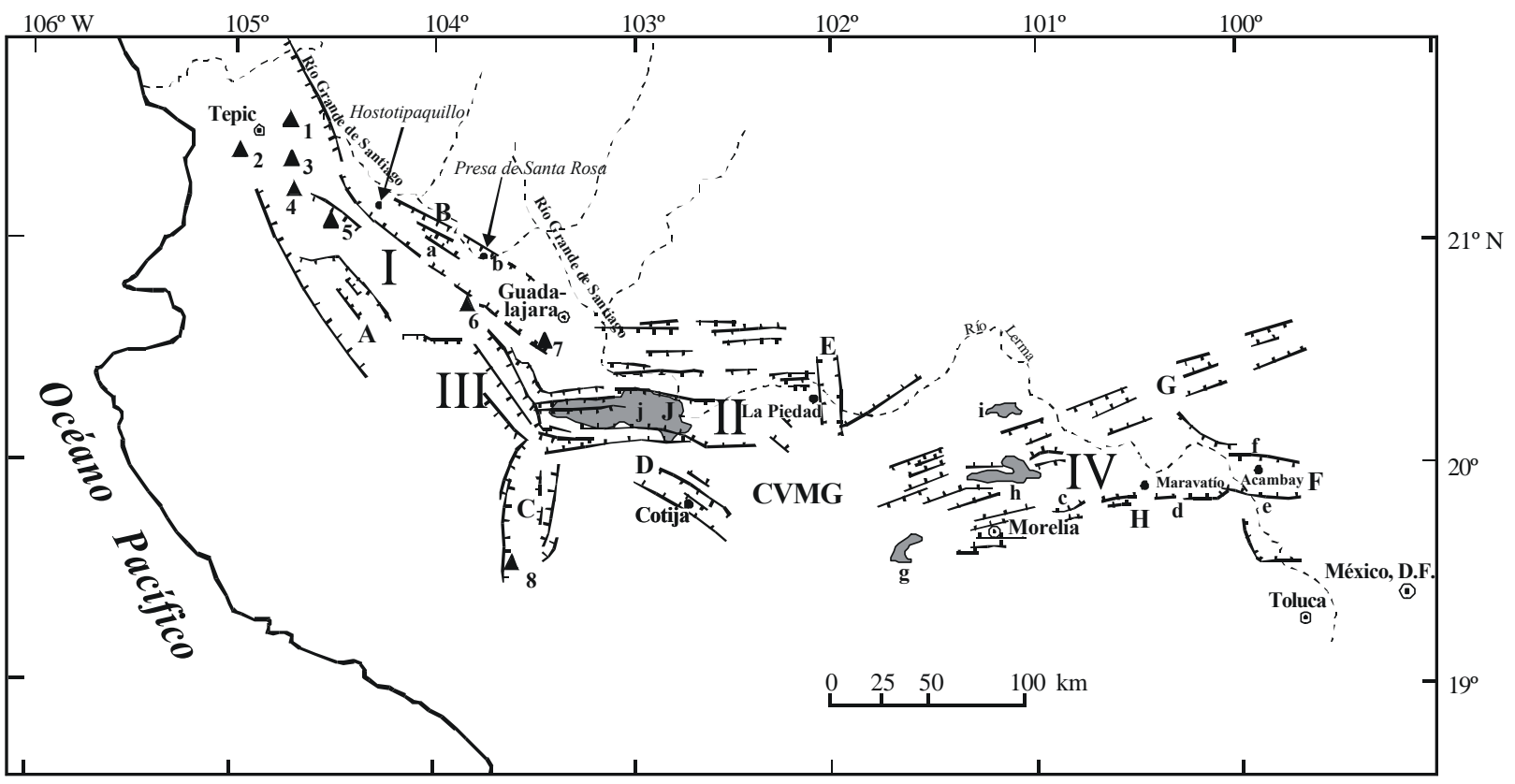

Figura 5. Marco tectónico regional de la porción centro-occidental de la Faja Volcánica Transmexicana. I: Rift Tepic-Chapala; II: Rift de Chapala; III: Graben de Zacoalco; IV: Zona de extensión Morelia-Acambay. Principales estructuras: A: Graben de Amatlán de Cañas; B: Graben de Plan de Barrancas-Tequila; C: Graben de Sayula; D: Graben de Cotija; E: Graben de Penjamillo; F: Graben de Acambay; G: Sistema de Fallas CuitzeoQuerétaro; H: Sistema de fallas Morelia-Acambay; I: Graben de Citala; J: Graben de Chapala. Fallas Principales: a: Falla de Plan de Barrancas; b: Falla Santa Rosa-Cinco Minas; c: Fallas de Morelia-Queréndaro-Los Azufres; d: Falla Venta de Bravo; e: Falla de Pastores; f: Falla de AcambayTixmadejé. Rasgos fisiográficos: g: Lago de Pátzcuaro; h: Laguna de Cuitzeo; i: Laguna de Yuriria; j: Lago de Chapala; 1: Volcán Las Navajas; 2: Volcán San Juan; 3: Volcán Sangangüey; 4: Volcán Tepeltiltic; 5: Volcán Ceboruco; 6: Volcán Tequila; 7: Caldera La Primavera; 8: Volcán de Colima; CVMG: Campo Volcánico Michoacán-Guanajuato. 
Tequila, Jal.), la pared meridional del cañón está formada por una secuencia de lavas basálticas del Mioceno tardío sobreyacida en discordancia angular por una intercalación de sedimentos lacustres con tobas e ignimbritas de 4.7 Ma (Nieto-Obregón et al., 1985). Una serie de coladas de lavas basálticas cuaternarias, que forma la Mesa de Santa Rosa, corona la secuencia.

Las lavas basálticas del Mioceno tardío presentan fracturas, fallas inversas y fallas de desplazamiento lateral, de dimensiones decimétricas, causadas por una contracción sub-meridiana (Figura 6, sitio MX93-87).

La población de fallas mostrada en la figura 6 (sitio MX93-87) muestra un solo juego cinemático de contracción, que se interpreta por la presencia de una sola dirección de estrías en los diferentes planos de falla de la población. Esto indica que las fallas no han sido retomadas posteriormente por algún otro campo de esfuerzos.

Al oeste de Hostotipaquillo, Jal. la secuencia igni mbrítica de la Sierra Madre Occidental se encuentra albierta en discordancia angular por productos volcánicos del Mioceno superior y del Plioceno. Las ignimbritas están inclinadas hacia el sur con echados que varían entre $\operatorname{los} 36^{\circ}$ y $\operatorname{los} 51^{\circ}$.

En esta secuencia ignimbrítica se midió una población de fallas inversas que también corresponde a un solo evento de deformación (sitio MX93-28). En los diferentes planos de falla se encuentran estrías con la misma dirección, lo que indica también que los planos de falla son resultado de un solo evento de deformación (Figura 6).

\section{Estructuras menores}

El análisis cualitativo de las estructuras menores que se observan sobre los planos de falla y en las zonas de falla, permite comparar los resultados obtenidos con el análisis de las estructuras mayores.

\subsection{Análisis cualitativo de las estructuras menores}

Las principales estructuras menores utilizadas en neotectónica son las grietas de tensión, las estilolitas y los planos de falla estriados. En general, podemos decir que los planos de las grietas de tensión son estadísticamente normales a la dirección principal de alargamiento, que los picos estilolíticos son estadísticamente paralelos a la dirección principal de acortamiento y que algunos tipos de estrías sobre los planos de falla permiten determinar el sentido de movimiento relativo de los dos bloques de una falla.

Se mostrará a continuación la utilización de las estructuras menores en neotectónica de poli-fases sobre un ejemplo de fallas en el Canal de Atalanti en Grecia, para ilustrar la interpretación de estructuras en una relación esfuerzo-deformación.

La figura 7A muestra un espejo de falla con surcos (estrías profundas) que corresponde a un movimiento de falla normal. Este espejo está deformado por un sistema de grietas de tensión, acomodadas en echelon, que materializa una junta de cizallamiento potencial en
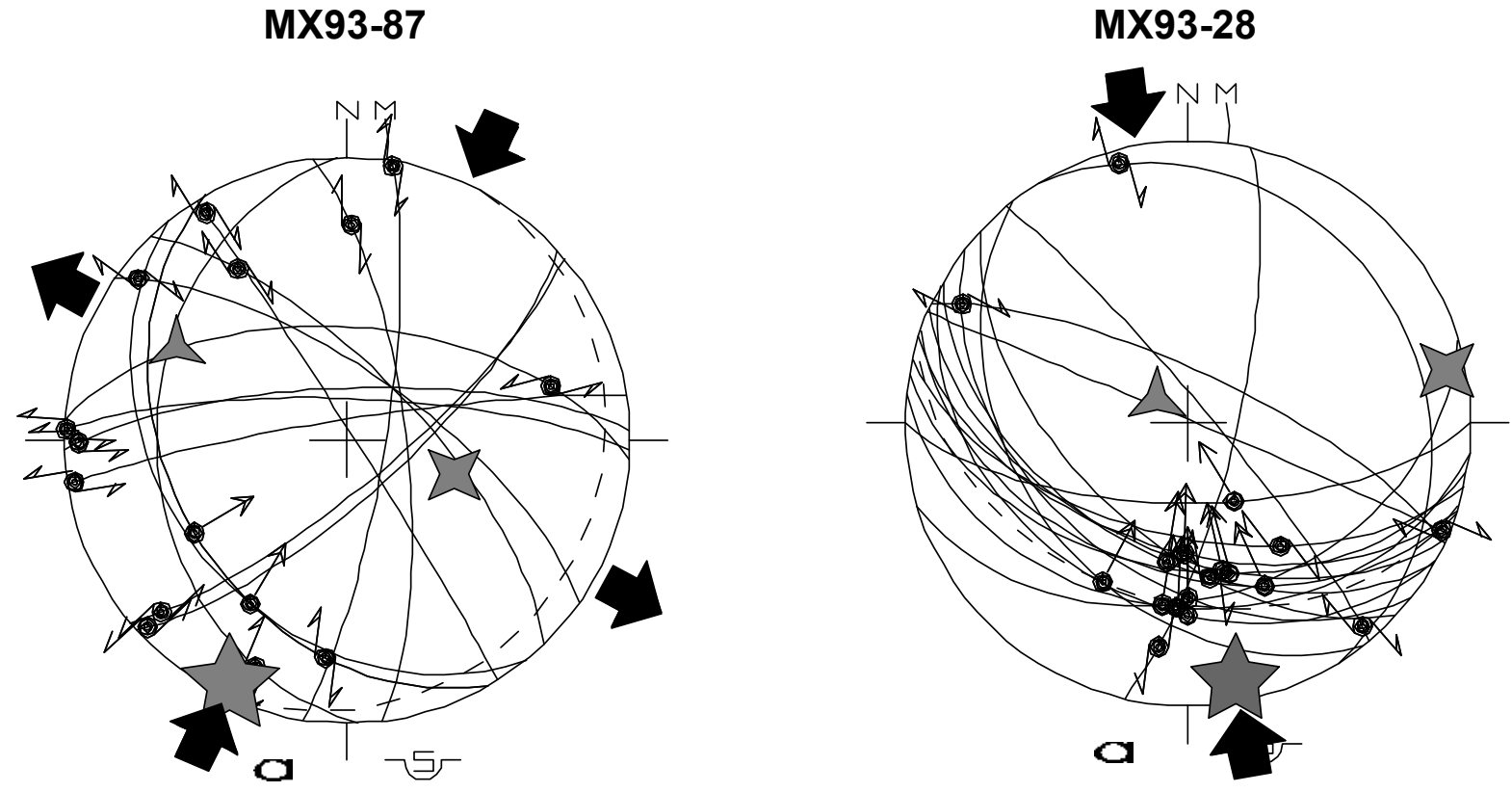

Figura 6. Diagramas estereográficos (equiareales) correspondientes al tensor de esfuerzos calculado, mostrando los planos de fallas de juego simple en contracción y sus polos así como sus estrías asociadas. Sitios localizados en la pared meridional del Cañón del Río Grande de Santiago en la Presa de Santa Rosa y de la región de Hostotipaquillo. 
falla inversa. Estas estructuras menores indican una deformación en extensión (1N) seguida de una deformación por cizalla (2I).

La Figura 7B muestra fracturas horizontales que fueron originadas por una deformación de acortamiento horizontal (tectónica de contracción), éstas están rellenas con minerales precipitados. Las fracturas están desplazadas por fallas menores cuyos espejos de falla contienen estrías que indican un movimiento de falla normal. Las estructuras menores testimonian una deformación de contracción (2I) seguida de una deformación de extensión $(3 \mathrm{~N})$.

La Figura 7C muestra un espejo de falla que porta tres familias de estrías que muestran surcos indicativos de movimiento de falla normal. Los surcos están pulidos por estrías estilolíticas que se formaron por el movimiento de bloques de una falla inversa. Las estrías estilolíticas son anteriores a otra familia de estrías muy finas que señalan un movimiento de falla normal. Estas estructuras menores son indicadores cinemáticos que atestiguan que esta falla ha tenido sucesivamente un movimiento de falla normal (1N) después desplazamiento de falla inversa (2I) y por último movimiento por una falla de tipo normal $(3 \mathrm{~N})$. Con el análisis de las estructuras menores es posible determinar la evolución cinemática en términos de paleoesfuerzos de una región que ha sufrido diferentes eventos de deformación.

El estudio de las estructuras menores de esta región en su conjunto, muestra siempre la misma sucesión: extensión-contracción-extensión, que se correlaciona positivamente con el estudio regional de estructuras mayores.

Existe una estrecha correlación entre los datos obtenidos para el estudio de estructuras grandes y pequeñas que permite validar las conclusiones en lo concerniente a la naturaleza de las deformaciones y a la
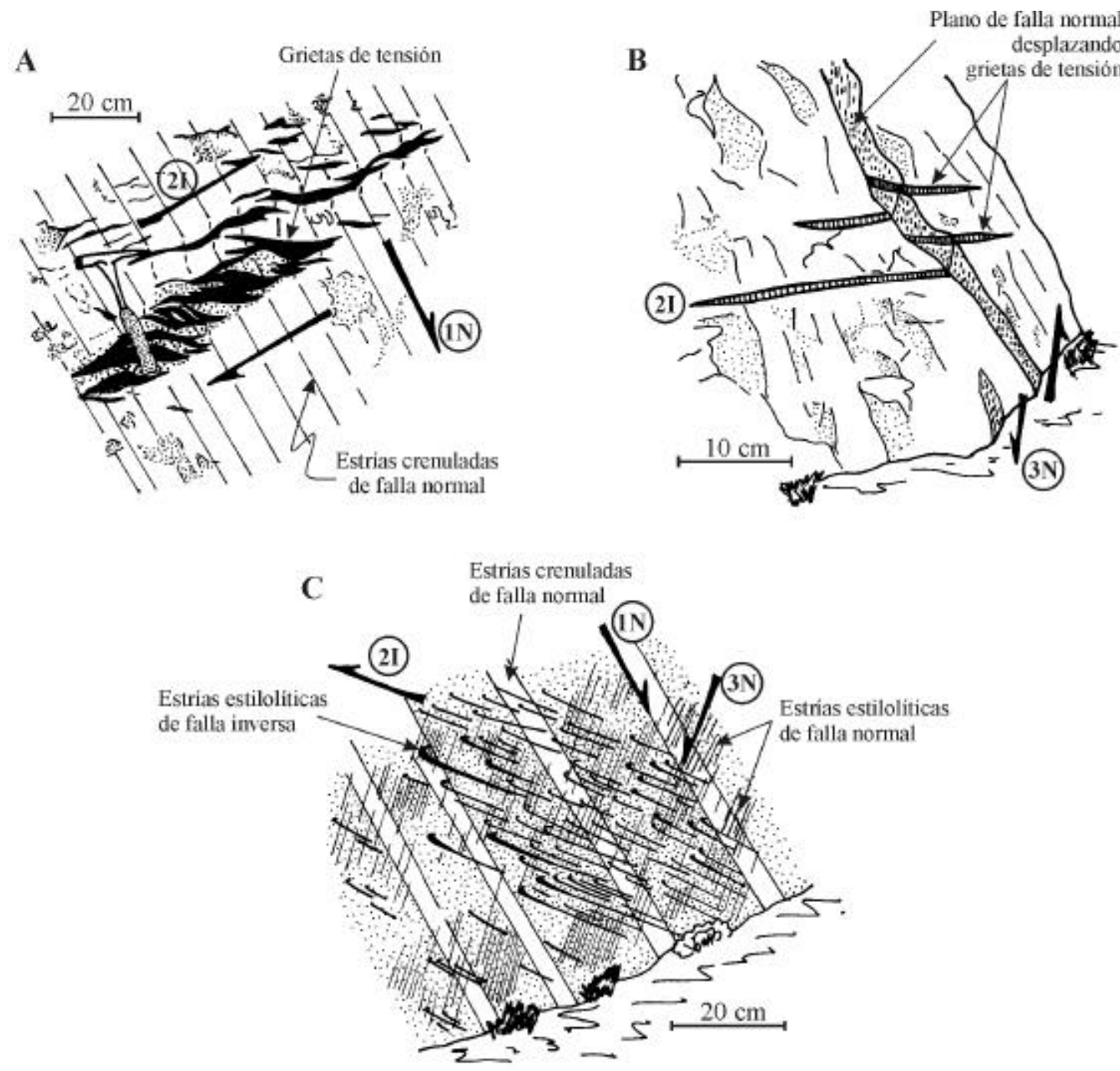

Figura 7. Análisis cualitativo de las estructuras menores en un plano de falla ubicado en el Canal de Atalanti en Grecia (modificado de Mercier, 1976). Ver texto para la explicación. 
sucesión de las diferentes fases de deformación, por lo que podemos trabajar con fallas grandes o pequeñas $y$ obtener las mismas conclusiones.

\section{Análisis cuantitativo de la deformación}

\subsection{El análisis de una población de fallas}

El análisis de una población de fallas se puede realizar utilizando la proyección estereográfica donde se proyectan los planos de fallas, las estrías y el sentido de movimiento. Si se cuenta con el movimiento de fallas independientes, se puede calcular un tensor de esfuerzos que se ajuste a la cinemática de ellas (Figura 8).

\subsection{Métodos gráficos}

\subsubsection{Método de los diedros agudos}

Con este método se determina la posición de los ejes principales de esfuerzo para un par de fallas conjugadas de desplazamiento lateral. Se trazan primero planos de falla, polos y estrías. A continuación se trazan los planos de movimiento uniendo estrías y polos de la misma falla con un círculo máximo; se definen entonces dos zonas (con líneas diagonales) con las intersecciones de los planos de falla y los planos de movimiento las cuales están formando un par de diedros agudos. Estos diedros agudos determinan la zona donde se localiza el eje del esfuerzo principal máximo $(\sigma 1)$, y su orientación general. Las flechas negras dirigidas hacia dentro indican

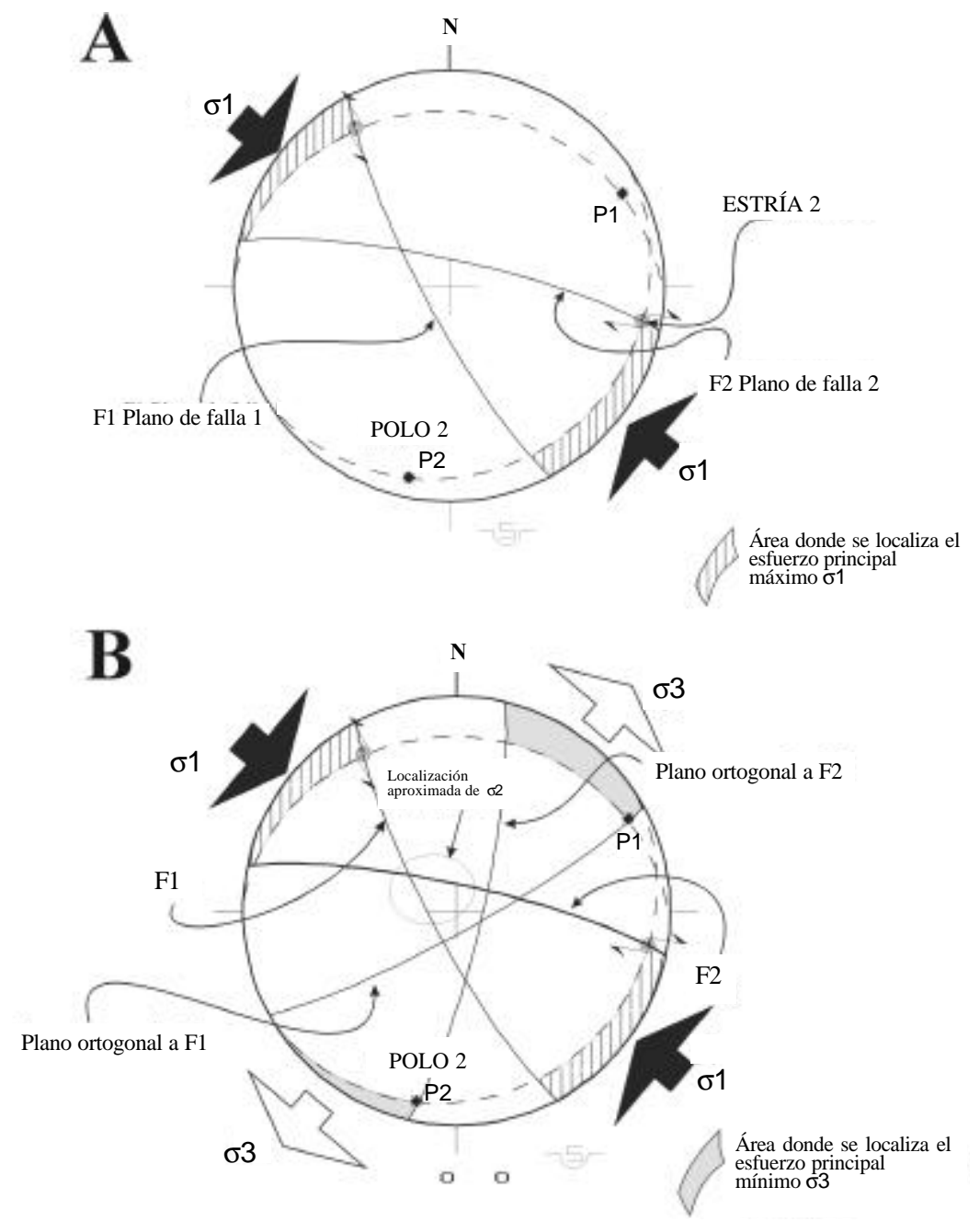

Figura 8. Determinación de la posición de los ejes principales de esfuerzo para un par de fallas conjugadas de desplazamiento lateral. Se trazan primero los planos de falla, polos y estrías. A continuación se trazan los planos de movimientos uniendo estrías y polos de la misma falla con un círculo máximo, se definen dos zonas, marcándolas con patrones de relleno, con las intersecciones de los planos de falla y los planos de movimiento los cuales están formando un par de diedros agudos. Estos diedros agudos determinan la zona donde se localizan el eje de esfuerzo principal mínimo $\left(\sigma_{1}\right)$, y su orientación general. Las flechas negras dirigidas hacia dentro indican una contracción orientada NW-SE. Ortogonalmente y en el plano horizontal o cercano a éste se localizará la posición del eje del esfuerzo principal mínimo $\left(\sigma_{3}\right)$ y ortogonalmente a los dos ejes de esfuerzo anteriores se localizará el eje de esfuerzo principal intermedio $\left(\sigma_{2}\right)$. Las flechas blancas dirigidas hacia fuera del círculo indican que existe una extensión orientada NE-SW. El eje del esfuerzo principal intermedio estará localizado aproximadamente en la zona de intersecciones de las fallas laterales derechas e izquierdas. 
Tabla 1. Ejemplos de datos obtenidos en planos de falla.

\begin{tabular}{|c|c|c|c|c|}
\hline No. & $\begin{array}{l}\text { Azimut del plano } \\
\text { de falla }\end{array}$ & $\begin{array}{l}\text { Inclinación del } \\
\text { plano de falla }\end{array}$ & Pitch de la estría & Juego del tipo de falla \\
\hline \multicolumn{5}{|l|}{ Sitio No. 1} \\
\hline 1 & $140^{\circ}$ & $90^{\circ}$ & $10^{\circ} \mathrm{N}$ & Lateral izquierda \\
\hline 2 & $152^{\circ}$ & $80^{\circ} \mathrm{W}$ & $16^{\circ} \mathrm{N}$ & Lateral izquierda \\
\hline 3 & $156^{\circ}$ & $80^{\circ} \mathrm{E}$ & $10^{\circ} \mathrm{S}$ & Lateral izquierda \\
\hline 4 & $119^{\circ}$ & $80^{\circ} \mathrm{S}$ & $05^{\circ} \mathrm{W}$ & Lateral derecha \\
\hline 5 & $112^{\circ}$ & $90^{\circ}$ & $19^{\circ} \mathrm{W}$ & Lateral derecha \\
\hline 6 & $102^{\circ}$ & $80^{\circ} \mathrm{N}$ & $10^{\circ} \mathrm{E}$ & Lateral derecha \\
\hline \multicolumn{5}{|l|}{ Sitio No. 2} \\
\hline 1 & $30^{\circ}$ & $60^{\circ} \mathrm{NW}$ & $80^{\circ} \mathrm{NE}$ & Normal \\
\hline 2 & $015^{\circ}$ & $50^{\circ} \mathrm{NW}$ & $80^{\circ} \mathrm{NE}$ & Normal \\
\hline 3 & $037^{\circ}$ & $66^{\circ} \mathrm{SE}$ & $80^{\circ} \mathrm{SE}$ & Normal \\
\hline 4 & $042^{\circ}$ & $60^{\circ} \mathrm{E}$ & $84^{\circ} \mathrm{S}$ & Normal \\
\hline \multicolumn{5}{|l|}{ Sitio No. 3} \\
\hline 1 & $121^{\circ}$ & $30^{\circ} \mathrm{S}$ & $178^{\circ} \mathrm{N}$ & Inversa \\
\hline 2 & $102^{\circ}$ & $40^{\circ} \mathrm{S}$ & $152^{\circ} \mathrm{N}$ & Inversa \\
\hline 3 & $074^{\circ}$ & $30^{\circ} \mathrm{N}$ & $005^{\circ} \mathrm{N}$ & Inversa \\
\hline 4 & $089^{\circ}$ & $20^{\circ} \mathrm{N}$ & $196^{\circ} \mathrm{N}$ & Inversa \\
\hline
\end{tabular}

una contracción orientada NW-SE (Figura 8B).

Ortogonalmente y en el plano horizontal o cercano a éste, se localiza la posición del eje del esfuerzo principal mínimo $(\sigma 3)$ y ortogonalmente a los dos ejes anteriores de esfuerzo se localizará el eje del esfuerzo principal intermedio $(\sigma 2)$. Las flechas blancas dirigidas hacia fuera del círculo indican que existe una extensión orientada NE-SW (Figura 8B). El eje de $\sigma 2$ estará localizado aproximadamente en la zona de intersección de las fallas laterales derechas e izquierdas.

Aplicación del método del diedro agudo. Los datos de los sitios No. 1, No. 2 y No. 3 de la Tabla 1 se pueden graficar en estereogramas para posteriormente interpretar por el método de diedro agudo las características de los esfuerzos principales que se ajusten a su cinemática. Las discontinuidades encontradas son juegos de fallas laterales.

\subsubsection{Método de los diedros rectos}

Este método consiste en sumar gráficamente los mecanismos focales de diferentes sismos de una misma región o localidad. En un diagrama estereográfico se dibujan sucesivamente los diedros de los mecanismos focales de tal manera que las áreas que corresponden a los diedros en tensión se muestran con diferente simbo- logía (con patrones de líneas horizontales, verticales, inclinadas, etc.) y las áreas en compresión se dejan en blanco.

Existirán entonces áreas comunes que definen diedros (en blanco y con múltiples patrones de relleno) donde se alojará el eje de tensión $\sigma 3$ o de compresión $\sigma 1$.

El método numérico del cálculo de un tensor medio para una población de fallas desarrollado por Angelier (1984, 1990) también es muy útil para caracterizar discontinuidades recientes, las cuales caen en el campo de la neotectónica.

\section{Conclusiones}

Los estudios de neotectónica permiten realizar análisis muy precisos de las deformaciones y de su cronología; análisis mucho más finos que aquellos que se obtienen para las deformaciones orogénicas más antiguas.

Desde un punto de vista general, los estudios de neotectónica deberían asegurar una transición instantánea entre los movimientos tectónicos -movimientos instantáneos (actividad sísmica)- formulados en términos de tectónica de placas y del estudio de episodios orogénicos más antiguos.

Desde el punto de vista de estudios regionales, los 
estudios de neotectónica permiten individualizar las deformaciones simples que se producen en un espacio de tiempo breve y ayudan a interpretar mejor las relaciones entre el esfuerzo y las deformaciones naturales, en particular las deformaciones frágiles de un medio discontinuo.

Desde un punto de vista más práctico, los estudios de neotectónica son necesarios para la interpretación correcta de perfiles sísmicos de reflexión en las márgenes continentales activas. Estos estudios son completamente indispensables para el análisis de sismos regionales, en particular para los trabajos de ingeniería civil en lugares con actividad sísmica frecuente.

\section{Referencias bibliográficas}

Angelier, J., 1984, Tectonic analysis of fault data sets: Journal of Ge ophysical Research, 89 (B7), 5835-5848.

Angelier, J., 1990, Inversion of field data in fault tectonics to obtain the regional stress; Part 3, A new rapid direct inversion method by analytical means: Geophysical Journal International, 103, 363376

Arreola, R., 1985, Obras completas de D. Melchor Ocampo. V. 1. La obra científica y literaria: Morelia, Comité Editorial del Gobierno del Estado de Michoacán, 510 p.

Astiz-Delgado, L.M., 1980, Sismicidad en Acambay, Estado de México. El temblor del 22 de febrero de 1979: México D.F., Universidad Nacional Autónoma de México, Facultad de Ingeniería, Tesis profesional, 130 p. (inédita).

Dobson, P.F., Mahood, G.A., 1985, Volcanic stratigraphy of the Los Azufres geothermal area, Mexico: Journal of Volcanology and Geothermal Research, 25, 273-287.
Mercier J.L., 1976, La néotectonique, ses méthodes et ses buts. Un exemple: l' arc égéen (Méditerranée orientale): Revue de Géographie Physique et de Géologie Dynamique, 18(4), 323346.

Nieto-Obregón, J., Delgado-Argote L.A., Damon, P.E., 1985, Geochronologic, petrologic, and structural data related to large morphologic features between the Sierra Madre Occidental and the Mexican Volcanic Belt: Geofísica Internacional, 24, 623-663.

Orozco y Berra, J.D., 1887, Efemérides sísmicas mexicanas: Memorias de la Sociedad Científica Antonio Alzate, 1, 303-541.

Ramírez, S., Reyes V., 1873, Informe sobre los temblores de Agua Fría y Jaripeo: Boletín de la Sociedad Mexicana de Geografía y Estadística, época 3, 1, 67-68.

Suter, M., Quintero-Legorreta, O., Johnson, C.A., 1992, Active faults and state of stress in the central part of the Trans-Mexican Volcanic Belt, México; 1, The Venta de Bravo Fault: Journal of Geophysical Research, 97, B8, 11983-11993.

Suter, M., López-Martínez, M., Quintero-Legorreta, O., CarrilloMartínez, M., 2001, Quaternary intra-arc extension in the central Trans-Mexican volcanic belt: Geological Society of America Bulletin, 113, 693-703.

Urbina, F., Camacho, H., 1913, La zona megasísmica AcambayTixmadejé, Estado de México, conmovida el 19 de noviembre de 1912: Boletín del Instituto Geológico de México, 32, 125 p.

Urquiza, M., 1872, Informe del ingeniero encargado de reconocer la Sierra de Ucareo para averiguar las probabilidades de una erupción volcánica: Boletín de la Sociedad Mexicana de Geografía y Estadística, época 2, 4, 586-588.

Manuscrito recibido: Agosto 12, 2000

Manuscrito aceptado: Julio 18, 2002 